\title{
Effect of Anti-Diabetic Treatment on High Density Lipoprotein- Composition and Lecithin: Cholesterol Acyltransferase Activity - A Comparison between Insulin, Sulfonylurea and Diet Alone Treatments
}

\author{
Takashi IIZUKA
}

\begin{abstract}
To determine the effect of diabetic treatment on plasma high density lipoprotein (HDL) composition and lecithin: cholesterol acyltransferase (LCAT) activity, 196 diabetic patients admitted to our hospital to receive diabetic control were divided into three groups according to the type of treatments; insulin treated group (91 patients), sulfonylurea group (56) and diet alone group (49). Plasma lipids, lipoproteins, apolipoproteins and LCAT activity were measured before and after the admission for 2-3 weeks. As compared with the pre-admission, the following results were obtained at the time of discharge in each group. 1) Body weight, fasting blood glucose, triglyceride, total cholesterol, apolipoproteins (A-I, A-II, C-II, C-III, E) were significantly decreased in each group. 2) Apoliprotein A-I/A-II ratio was significantly increased from 4.9 to 5.3 in insulin treated group $(\mathbf{p}<0.001)$. 3) Total HDL-cholesterol was significantly decreased in diet alone and sulfonylurea groups, however HDL 3-cholesterol was significantly decreased in sulfonylurea and insulin group. Then HDL 2-cholesterol/HDL 3-cholesterol ratio was significantly increased from 4.8 to 5.1 (p $<0.001)$ in insulin group. 4) LCAT activity were significantly decreased from 790 to $637(\mathrm{nmol} / \mathrm{ml} \cdot \mathrm{h})(\mathrm{p}<0.05)$ in insulin group, but did not change in other two groups. In conclusion, compared to diet alone and sulfonylurea groups, insulin group made significant reduction of HDL 3-cholesterol and LCAT activity during hospitalization.
\end{abstract}

Key Words: High density lipoprotein, Lecithin: cholesterol acyltransferase activity, Diabetes mellitus

It is generally accepted that improved glucoregulation will result in normalization of elevated lipid levels (1-7). However, the effect of diabetic control on plasma high density lipoprotein (HDL) levels and lecithin: cholesterol acyltransferase (LCAT) activity, which play an important role of anti-atherogenic metabolism, is not fully examined. Although metabolic control leads to a reduction in triglyceride and triglyceride-rich lipoproteins, its effect of HDL and LCAT activity responses to glycemic control in diabetic patients might differ in different treatments. Therefore, we examined the effect of 2-3 weeks of hospitalization on plasma lipids, apolipoproteins, HDL-compositions and LCAT activity levels in 196 diabetic patients.

\section{PATIENTS AND METHODS}

Patients: All 196 diabetic patients who were ad- mitted to the Institute for Diabetes Care and Research, Asahi Life Foundation were studied. Ninety-one of these patients were on insulin, 56 on sulfonylurea and 49 on diet alone therapy.

The mean age was $52 \pm 15$ yr for insulin group, $59 \pm 19$ yr for sulfonylurea group and $54 \pm 8$ yr for diet alone group (Mean $\pm \mathrm{SD}$ ), respectively. Seventy of these patients had retinopathy, while 36 had persistant proteinuria (nephropathy) and 81 had neuropathy. Table 1 summaries a clinical and metabolic characteristics of all these patients.

All patients were placed on an eucalolic diet of 20-30 Kcal. per standard body weight [(height$100) \times 0.9$ ] $(\mathrm{kg})$ consising of $20 \%$ calories as protein, $26 \%$ as fat, $46 \%$ as carbohydrate and $8 \%$ others. Their activity was maintained as constant as possible during hospitalization. Regular exercise schedules were used in all patients during hospitalization. The

From The Institute for Diabetes Care and Research, Asahi Life Foundation, Tokyo

Received for publication November 25, 1988.

Reprint request to: Takashi Iizuka, MD, The Institute for Diabetes Care and Research, Asahi Life Foundation, 1-6-1 Marunouchi, Chiyoda-ku, Tokyo 100, Japan 
Table 1. Clinical treatments and metabolic characteristics of diabetic patients with different treatments.

\begin{tabular}{lrrr}
\hline & Insulin & \multicolumn{1}{c}{$\begin{array}{l}\text { Sulfonyl } \\
\text { urea }\end{array}$} & \multicolumn{1}{c}{ Diet } \\
\hline $\begin{array}{l}\text { Number } \\
\text { (male/female) }\end{array}$ & $\begin{array}{c}91 \\
(54 / 37)\end{array}$ & $\begin{array}{c}56 \\
(34 / 22)\end{array}$ & \multicolumn{1}{c}{$\begin{array}{c}49 \\
(44 / 5)\end{array}$} \\
& 52.0 & 59.0 & 53.0 \\
Age (years) & \pm 15.3 & \pm 10.1 & \pm 8.3 \\
& 103.0 & 108.8 & 119.5 \\
Relative body & \pm 13.4 & \pm 14.5 & \pm 22.0 \\
weight (\%)* & 10.1 & 9.7 & 8.1 \\
& \pm 2.2 & \pm 1.8 & \pm 1.4 \\
HbA c (\%) & 13.2 & 11.3 & 6.4 \\
& \pm 8.1 & \pm 6.3 & \pm 5.8 \\
Duration (years) & 22.2 & 14.9 & 12.1 \\
& \pm 20.4 & \pm 7.5 & \pm 9.2 \\
Period of & 30.4 & 63.4 & 63.2 \\
admission (days) & \pm 26.8 & \pm 35.8 & \pm 44.1 \\
\hline Urinary CPR & &
\end{tabular}

* relative body weight $(\%)=\frac{(\text { body weight }) \times 100(\%)}{(\text { height }-100) \times 0.9}$ Mean $\pm S D$

schedules of daily diet and activity was maintained as close to prehospital advices for all patients as possible.

None of them had evidence of liver disease, nephrotic syndrome, hypothyroidism or other disorders frequently associated with increased blood lipid levels. None of the medications affecting blood lipid levels had been used since pre-admission.

Twenty patients $(10 \%)$ were taking medications other than insulin and sulfonylurea, including diuretics, calcium antagonists and angiotensin converting enzyme inhibitors. These medications were not change during this study.

The mean period of hospitalization was 22 days in insulin group, 15 days in sulfonylurea group and 12 days in diet alone group.

Methods: Blood was drawn after an overnight fast. Plasma for LCAT assay was obtained from unselected diabetic patients; twenty six patients with insulin, 20 patients with sulfonylurea and 20 patients with diet alone therapy and one aliquot was immediately frosen at $-80^{\circ} \mathrm{C}$.

Triglyceride and total cholesterol were measured with enzymatic methods, HDL cholesterol was measured with heparin manganese methods.

HbAlc was measured by HPLC methods, its normal levels in our laboratory ranged from $4 \%$ to $6.5 \%$. Apolipoproteins (A-I, A-II, B, C-II, C-III, E) were measured by SRID methods. The normal mean values reported by Goto et al. (8) were 135 , $32,79,3.4,7.5,4.1 \mathrm{mg} / \mathrm{dl}$, respectively.

The separation of HDL $2(\mathrm{~d}=1.063-1.125)$ and HDL3 $(\mathrm{d}=1.123-1.210)$ was performed by ultracentrifugation on a preparative ultracentrifuge (Beckman L 15-50) using a fixed angled rotor (type 50) with appropriate adaptors. The plasma density was adjusted to $\mathrm{d}=1.063 \mathrm{~g} / \mathrm{ml}$ with $\mathrm{KBr}$ solution and separated at $4^{\circ} \mathrm{C}$ by 20 -hours centrifugation at $40,000 \mathrm{rpm}$. After centrifugation, the $2 / 3$ infranate was adjusted to $\mathrm{d}=1.125 \mathrm{~g} / \mathrm{ml}$ with $\mathrm{KBr}$ solution and was centrifugated at $4^{\circ} \mathrm{C}-40,000 \mathrm{rpm}$ for 40 hours. After the second centrifugation, the plasma$\mathrm{KBr}$ mixture tube was sliced in the middle of the clear zone. Both the supranate (HDL 2) and the infranate (HDL 3) were dialyzed exhaustively 3 -times changes of $0.9 \% \mathrm{NaCl}$ solution at $4^{\circ} \mathrm{C}$. The cholesterol and triglyceride of two HDL fractions were measured by enzymatic methods.

LCAT activity was measured according to the following method reported by Takahashi et al. (9).

1) Preparation of substrate vesicles: $\left(7 \alpha{ }^{3} \mathrm{H}\right)$ cholesterol and unesterified cholesterol were well mixtured and purified by thin layer chromatography. The mixture in addition to egg-phosphatidylcholine was dissolved in ethanol and was dialyzed exhausedly 3-times changes against phosphate buffer $\left(\mathrm{KH}_{2} \mathrm{PO}_{4}-\mathrm{Na}_{2} \mathrm{HPO}_{4}\right)$ at $4^{\circ} \mathrm{C}$ to remove ethanol. The single bilayer vesicles of egg-phosphatidylcholine and $\left(7 \alpha-{ }^{3} \mathrm{H}\right)$ cholesterol mixture in addition to $0.1 \mathrm{M}$-sodium cholate solution were incubated in the presence of purified apolipoprotein A-I for 30 minutes at $37^{\circ} \mathrm{C}$, and then dialyzed exhaustly 5 -times changes against phosphate buffer at $4^{\circ} \mathrm{C}$.

2) The measurement of LCAT activity: The assay mixture consisted of $100 \mu \mathrm{l}$ of vasicle solution (90 nmol of egg-phosphatidylcholine and $15 \mathrm{nmol}$ of $\left(7 \alpha-{ }^{3} \mathrm{H}\right)$ cholesterol, specific activity 4 $\mathrm{mCi} / \mathrm{mmol}), 15 \mu \mathrm{g}$ of apolipoprotein A-I, $4 \mathrm{mM}$ 2-mercaptoethanol, $0.7 \mathrm{mM}$ EDTA, $2.5 \mathrm{mg}$ of bovine serum albumin and $1 \mu \mathrm{l}$ of the plasma sample diluted to a final volume of $10 \mu$ with phosphate buffer. The assay mixtures flushed with $\mathrm{N}_{2}$ were incubated for 1 hour at $37^{\circ} \mathrm{C}$ with mechanical shaking. 
After incubation, $3 \mathrm{ml}$ of chloroform: methanol $(1: 1, \mathrm{v} / \mathrm{v})$ was added to the mixture in order to stop this reaction. The radioactivity in the cholesterol and cholesterol-ester fractions separated by thin layer chromatography was measured by a liquid scintillation counter. LCAT activity was calculated by the following equations:

1) Cholesterol esterification rate $=\mathrm{EC} /(\mathrm{FC}+$ EC) $\times 100(\%)$

2) LCAT activity $=15(\mathrm{nmol}) \times \mathrm{EC} /(\mathrm{FC}+$ EC) $\times 1 / 1 \times 1,000(\mu \mathrm{l}) / 1(\mu \mathrm{l})$

[EC: cholesterol ester, FC: free cholesterol]

$(\mathrm{nmol} / \mathrm{ml} \cdot \mathrm{h})$

Under these assay condition, the rate of cholesterol esterification was linear and ranged from $5 \%$ to $25 \%$ during 1-hour incubation period.

Results were expressed as the mean \pm the standard deviation. The significance of differences was assessed using a paired t-test. Only values with $\mathrm{p}<0.05$ were regarded as significant.

\section{RESULTS}

Table 2 summarizes the laboratory data in all patients studied on admission and at discharge from the hospital.

\section{1) Plasma lipids and the degree of diabetic control}

Body weight, fasting blood glucose, plasma triglyceride and total cholesterol were significantly decreased, but plasma LDL cholesterol did not change.

\section{2) Plasma apolipoproteins}

Plasma apolipoproteins (A-I, A-II, B, C-II, C-III, E) were significantly decreased except for apolipoprotein B in diet alone group. However, apolipoprotein A-I/A-II ratio was significantly increased from 4.9 to $5.3(\mathrm{p}<0.001)$ in insulin group.

\section{3) Plasma HDL-cholesterol, cholesterol and triglyceride levels of HDL subfractions}

Table 2. Body weight, fasting blood glucose, fasting plasma lipids, lipoproteins, apolipoproteins, HDL-subtraction cholesterol, triglyceride, LCAT activity in the diabetic patients at admission and discharge from the hospital.

\begin{tabular}{|c|c|c|c|c|c|c|}
\hline & \multicolumn{2}{|c|}{$\begin{array}{l}\text { Patients treated by } \\
\text { diet alone, } \mathrm{n}=49\end{array}$} & \multicolumn{2}{|c|}{$\begin{array}{l}\text { Patients treated by } \\
\text { sulfonylurea, } n=56\end{array}$} & \multicolumn{2}{|c|}{$\begin{array}{l}\text { Patients treated by } \\
\text { insulin, } n=91\end{array}$} \\
\hline & at admission & at discharge & at admission & at discharge & at admission & at discharge \\
\hline Body weight (kg) & $70.3 \pm 13.8$ & $67.6 \pm 12.5^{(* * *)}$ & $57.0 \pm 13.2$ & $55.0 \pm 12.7(* * *)$ & $56.1 \pm 8.5$ & $54.2 \pm 8.0(* * *)$ \\
\hline Fasting blood glucose (mg/dl) & $129.1 \pm 28.2$ & $100.3 \pm 17.4(* * *)$ & $161.2 \pm 57.3$ & $103.7 \pm 27.2(* * *)$ & $160.9 \pm 64.9$ & $118.3 \pm 54.2(* * *)$ \\
\hline Total cholesterol (mg/dl) & $204.4 \pm 41.3$ & $189.0 \pm 35.4(* * *)$ & $206.0 \pm 41.9$ & $\left.189.8 \pm 38.3^{* * *}\right)$ & $211.2 \pm 62.6$ & $198.6 \pm 55.8\left(^{* * *}\right)$ \\
\hline Triglyceride (mg/dl) & $162.6 \pm 130.8$ & $115.8 \pm 79.6(* * *)$ & $169.3 \pm 162.7$ & $105.6 \pm 54.5\left(^{* * *}\right)$ & $110.9 \pm 59.4$ & $92.0 \pm 55.4(* *)$ \\
\hline HDL-cholesterol (mg/dl) & $45.3 \pm 13.3$ & $42.6 \pm 10.3\left(^{*}\right)$ & $47.4 \pm 15.5$ & $44.3 \pm 12.5(* *)$ & $50.7 \pm 14.7$ & $49.7 \pm 13.3$ \\
\hline LDL-cholesterol (mg/dl) & $128.1 \pm 41.0$ & $123.8 \pm 35.5$ & $126.2 \pm 44.1$ & $125.2 \pm 35.4$ & $123.8 \pm 46.6$ & $121.3 \pm 43.1$ \\
\hline Apolipoprotein A-I (mg/dl) & $118.8 \pm 19.8$ & $109.8 \pm 21.6\left(^{*}\right)$ & $122.9 \pm 26.5$ & $109.9 \pm 21.5(* * *)$ & $118.3 \pm 19.9$ & $111.3 \pm 18.7(* *)$ \\
\hline A-II $(\mathrm{mg} / \mathrm{dl})$ & $27.6 \pm 5.9$ & $25.2 \pm 5.1(* *)$ & $27.7 \pm 6.1$ & $24.4 \pm 5.1(* * *)$ & $24.9 \pm 5.2$ & $22.4 \pm 4.4(* * *)$ \\
\hline$B(\mathrm{mg} / \mathrm{dl})$ & $106.9 \pm 28.9$ & $102.0 \pm 23.6$ & $104.9 \pm 24.7$ & $100.0 \pm 23.5\left(^{*}\right)$ & $105.1 \pm 38.9$ & $99.5 \pm 32.4(* *)$ \\
\hline C-II $(\mathrm{mg} / \mathrm{dl})$ & $4.6 \pm 2.1$ & 4. $0 \pm 1.5\left(^{*}\right)$ & $5.0 \pm 3.1$ & $4.1 \pm 1.5(* *)$ & 4. $0 \pm 1.7$ & $3.5 \pm 1.4(* *)$ \\
\hline C-III $(\mathrm{mg} / \mathrm{dl})$ & $9.1 \pm 4.3$ & $6.9 \pm 2.4(* * *)$ & $10.6 \pm 6.9$ & $7.6 \pm 3.2(* * *)$ & $8.4 \pm 3.7$ & $7.2 \pm 3.2(* * *)$ \\
\hline$E(\mathrm{mg} / \mathrm{dl})$ & $4.5 \pm 1.6$ & $3.8 \pm 1.2(* * *)$ & $4.6 \pm 2.0$ & $3.6 \pm 1.0(* * *)$ & $3.9 \pm 1.2$ & $3.5 \pm 1.0(* * *)$ \\
\hline A-I/A-II ratio & $4.42 \pm 0.79$ & $4.48 \pm 0.65$ & $4.50 \pm 0.70$ & $4.60 \pm 0.80$ & $4.84 \pm 0.77$ & $5.08 \pm 0.93(* * *)$ \\
\hline HDL2-cholesterol ( $\mathrm{mg} / \mathrm{dl})$ & $19.9 \pm 9.3$ & $19.3 \pm 7.9$ & $19.3 \pm 7.7$ & $19.8 \pm 8.3$ & $27.1 \pm 12.6$ & $28.4 \pm 11.4$ \\
\hline HDL2-triglyceride (mg/dl) & $18.1 \pm 5.5$ & $18.6 \pm 6.9$ & $16.6 \pm 5.6$ & $17.3 \pm 4.2$ & $15.8 \pm 4.6$ & $16.8 \pm 4.7$ \\
\hline HDL3-cholesterol (mg/dl) & $24.0 \pm 7.3$ & $22.9 \pm 6.4$ & $26.8 \pm 7.0$ & $22.1 \pm 4.9\left(^{*}\right)$ & $30.5 \pm 9.7$ & $23.1 \pm 7.2(* * *)$ \\
\hline HDL3-triglyceride (mg/dl) & $16.3 \pm 7.6$ & $16.8 \pm 7.2$ & $17.0 \pm 8.1$ & $14.4 \pm 2.7$ & $13.6 \pm 4.9$ & $12.9 \pm 3.6$ \\
\hline HDL2-ch /HDL3-ch. ratio & $0.83 \pm 0.45$ & $0.83 \pm 0.37$ & $0.74 \pm 0.26$ & $0.96 \pm 0.45$ & $0.91 \pm 0.45$ & $1.30 \pm 0.57(* * *)$ \\
\hline HCL2-TG/HDL3-TG ratio & $1.19 \pm 0.24$ & $1.19 \pm 0.25$ & $1.02 \pm 0.21$ & $1.19 \pm 0.22(*)$ & $1.20 \pm 0.27$ & $1.29 \pm 0.56$ \\
\hline LCAT activity (nmoles/ml hour) & $645.1 \pm 310.9$ & $584.5 \pm 249.3$ & $537.1 \pm 173.1$ & $509.0 \pm 186.8$ & $790.0 \pm 362.8$ & $636.8 \pm 208.2(*)$ \\
\hline Insulin (Unit) & & & & & $20.1 \pm 10.1$ & $15.7 \pm 7.4(* * *)$ \\
\hline
\end{tabular}

$* \mathrm{p}<0.05, \quad * * \mathrm{p}<0.01, \quad * * * \mathrm{p}<0.001 . \quad$ Mean $\pm \mathrm{SD}$ 
Plasma HDL-cholesterol in sulfonyluea and diet alone groups was significantly decreased, although no significant change was observed in insulin group. Plasma HDL 2-cholesterol did not change in each group, but plasma HDL 3-cholesterol was significantly decreased in sulfonylurea and insulin group. However, plasma HDL 2-cholesterol/HDL 3 -cholesterol ratio was significantly increased from 0.9 to $1.3(p<0.001)$ in insulin group. Plasma HDL 2-, HDL 3-triglyceride did not change in any groups, but plasma HDL 2-triglyceride/HDL 3 triglyceride ratio was significantly increased in sulfonylurea group.

\section{4) Plasma LCAT activity}

Plasma LCAT activity was measured in unselected sixty-six patients; 26 patients with insulin, 20 patients with sulfonylurea and 20 patients with diet alone treatment. Plasma LCAT activity was significantly decreased from 790 to 637 $(\mathrm{nmol} / \mathrm{ml} \cdot \mathrm{h})$ in insulin group, but did not change in other two groups.

\section{DISCUSSION}

Accelerated atherosclerosis, a common clinical problem in diabetic patients, appears to be at least partly related to altered lipid and lipoprotein metabolism in diabetics. Previous studies examining the effect of diabetic control on plasma lipid levels have found that improved glucoregulation results in a reduction of elevated lipid levels. More recently, attention has been focused on the effect of diabetic treatment on lipoprotein concentrations $(10,11)$. It is well known that uncontrolled diabetes often results in elevation of TG and TG-rich lipoprotein levels, but less is known of the effect of diabetes on plasma HDL and LCAT activity levels. Knowledge of the effect of diabetic control on these latter two is particularly important.

Thus, we examined the effect on plasma HDLcompositions (HDL 2 and HDL 3) and LCAT activity in different three treatments before and after 2-3 weeks hospitalization, which is probably able to keep on strict control in diet and exercise treatment.

Until now, Agardh et al. (1) reported increased levels of plasma HDL cholesterol after 3-4 months' insulin therapy in 26 non-obese diabetic patients, while Lopes-Virella et al. (2) reported a significant increase of plasma HDL-cholesterol in 20 male diabetics and no change in 35 female diabetics after 2-3 week hospitalization with intensive insulin therapy. Further, Yasuoka et al. (3) reported a significant increase of plasma HDL-cholesterol and HDL 2-cholesterol associated with no change of plasma HDL 3-cholesterol and LCAT activity in insulin treated diabetics after 2 months' diabetic control. However, few investigators reported the effect of hospitalization, which probably leads to strict diabetic control, on plasma HDL-composition and LCAT activity in different treatment. The effect of diet and exercise on plasma lipid and lipoprotein levels observed during hospitalization cannot be excluded. We think, however, that this is an unlikely possibility since all patients were followed regularly by us in an outpatient clinic and they have been previously on a similar dietary plan and treatments. With same diet and exercise, the different results among the three groups probably show the therapeutic effects on plasma HDL-composition and LCAT activity.

Our results demonstrated the therapeutic difference on plasma HDL-cholesterol, HDL composition and LCAT activity; plasma HDL 2cholesterol/HDL 3-cholesterol ratio was significantly increased from 0.9 to 1.3 and plasma LCAT activity was significantly decreased from 790 to 637 $(\mathrm{nmol} / \mathrm{ml} \cdot \mathrm{h})$ in insulin group.

Nikkilä (12) reported that peripheral hyperinsulinemia induced by insulin treatment in diabetics resulted in an increase of tissue lipoprotein lipase activity which brought about increase of plasma HDL-cholesterol, particularly HDL 2-cholesterol. According to Nikkilä (12), the increase of plasma HDL-cholesterol in those previous reports is probably dependent on existence of peripheral hyperinsulinemia induced by the acute change for insulin therapy. However, because of this significant decrease of average insulin dose, it appears that plasma HDL-cholesterol and HDL 2-cholesterol may not increase in our study.

Pietri et al. (4) and Dunn et al. (5) reported that plasma HDL-cholesterol levels did not rise until 2 months of continous subcutaneous insulin infusion therapy, although TG-rich lipoprotein levels were significantly decreased after 2-3 weeks of both 
conventional insulin therapy and continuous subcutaneous insulin infusion therapy. However in our study, plasma HDL-cholesterol and HDL 3cholesterol were significantly dechanged following 2-3 week hospitalization. Thus, we speculate that glucoregulation by each treatment causes a significant decrease of plasma HDL as well as TG-rich lipoprotein in 2-3 week hospitalization.

As for the correlation between plasma apolipoproteins and HDL compositions, Eckel et al. (13) reported the high levels of plasma apolipoprotein A-I/A-II ratio and HDL 2-cholesterol in insulin dependent diabetics. In our study, both plasma HDL 2-cholesterol and apolipoprotein A-I/A-II ratio were highest in insulin treated group. Further, Yachida and Minari (14) reported that $78 \%$ of HDL 2-apolipoprotein was (A-I) type, $72 \%$ of HDL 3-apolipoprotein was (A-I) 2 (A-II) $)_{2}$ type. According to Yachida and Minari (14), we can understand that the decreases of apolipoproteins A-I and A-II correspond to the decrease of HDL 3-cholesterol in sulfonylurea and insulin groups. Furthermore, the significant increase of both apolipoprotein A-I/AII ratio and HDL 2-cholesterol/HDL 3-cholesterol ratio in insulin-treated group can be understood.

The mechanism of the significant decrease of plasma LCAT activity in insulin group is not clear. However Akanuma et al. (15) reported that serum LCAT activity was significantly correlated with increases in relative body weight, serum total cholesterol, serum unesterified cholesterol, serum basal immunoreactive insulin and serum triglyceride. Therefore, in our study, the significant decrease of plasma LCAT activity in insulin group may result from changes of plasma lipid and insulin levels.

ACKNOWLEDGEMENTS: The auther would like to thank Dr. Y. Akanuma in the Institute for Diabetes Care and Research, Asahi Life Foundation and Prof. K. Murata in the Second Department of Internal Medicine, Gunma University, for their helpful advice of this study.

\section{REFERENCES}

1) Agardh CD, Nilsson Ehle $P$, Schersten $B$ : Improvement of the plasma lipoprotein pattern after institution of insulin treatment in diabetes mellitus. Diabetes Care 5: 322, 1982.

2) Lopes-Virella MFL, Wohltmann HJ, Mayfield RK, Loadholt CB, Colwell JA: Effect of metabolic control on lipid, lipoprotein and apolipoprotein level in 55 insulin dependent diabetic patients, a longitudinal study. Diabetes 32: 20, 1983.

3) Yasuoka N, Kawada M, Kishino W, et al: Changes on serum HDL2,3 cholesterol after improved glycemic control in diabetics with different treatment. J Jpn Atheroscler Soc 13: 393, 1985.

4) Pietri A, Dunn FL, Raskin P: The effect of diabetic control on plasma lipid and lipoprotein levels: A comparison of conventional therapy and continuous subcutaneous insulin infusion. Diabetes 29: 1001, 1980.

5) Dunn FL, Pietri A, Raskin P: Plasma lipid and lipoprotein level with continuous subcutaneous insulin infusion in type I diabetes melitus. Ann Intern Med 95: 426, 1981.

6) Iizuka T: Effect of diabetic control on high-densitylipoprotein composition and lecithin: cholesterol acyltransferase activity in diabetic patients on insulin treatment. J Jpn Diabetes Soc 31: 41, 1988.

7) Iizuka $T$ : Effect of strict diabetic control on serum lipid and HDL-LCAT metabolism during hospitalization. A study of non-insulin-dependent-diabetic patients with sulfonylurea treatment. J Kitakanto Med Soc 39: 267, 1989.

8) Goto Y, Akanuma Y, Harano Y, et al: Determination by the SRID method of normal values of serum apolipoproteins (A-I, A-II, B, C-II, C-III, and E) in normolipidemic healthy Japanese subjects. J Clin Biochem Nutr 1: 73, 1986.

9) Takahashi K, Murase T, Akanuma Y: Measurement of lecithin; cholesterol acyltransferase activity. "Handbook of biochemical experiments; 1-membrane lipid and plasma lipoprotein”' Tokyo, Jpn Biochem Soc 10, 1986.

10) West KM, Ahuja MM, Bannett $\mathrm{PH}$, et al: The role of circulating glucose and triglyceride concentrations and interactions with other 'risk factors' as determinants of arterial disease in nine diabetic population samples from the WHO multinational study. Diabetes Care 6: $361,1983$.

11) Gordon T, Castelli WP, Hjortland MC, Dawber TT: Diabetes, blood lipids and the role of obesity in coronary heart disease risk for women. The Framingham Study. Ann Intern Med 87: 393, 1977.

12) Nikkilä EA: High density lipoprotein in diabetes. Diabetes (Supple 2) 30: 82, 87, 1981.

13) Eckel RH, Albers JJ, Cheung MC, Wahl PW, Lindgren FT and Bierman EL: High density lipoprotein composition in insulin dependent diabetes mellitus. Diabetes 30: 132, 1981.

14) Yachida $Y$, Minari O.: Studies on human plasma HDL 2 and HDL 3 by various bifunctional cross linking reagents (III). Functional and purification of HDL of $\mathrm{A} \mathrm{I}_{3} \mathrm{C}_{2}$ type. J Lib Arts Sci Sappro Med Coll 22: 57, 1981.

15) Akanuma $Y$, Kuzuya T, Hayashi M, Ide T, Kuzuya N: Positive correlation of serum lecithin: cholesterol acyltransferase activity with relative body weight. Eur J Clin Invest 3: 136, 1972. 